\title{
ON THE MEAN-VALUE THEOREM CORRESPONDING TO A GIVEN LINEAR HOMOGENEOUS DIFFERENTIAL EQUATION *
}

\author{
BY \\ G. PÓLYA
}

I consider the linear homogeneous differential expression of order $n$ $L f(x) \equiv f^{(n)}(x)+\phi_{1}(x) f^{(n-1)}(x)+\phi_{2}(x) f^{(n-2)}(x)+\cdots+\phi_{n}(x) f(x)$. I suppose $\phi_{1}(x), \phi_{2}(x), \cdots, \phi_{n}(x)$ are continuous, $f(x)$ is differentiable $n$ times, the variable $x$ and all the functions in question are real. I then prove some theorems concerning the equation

$$
L y \equiv \frac{d^{n} y}{d x^{n}}+\phi_{1} \frac{d^{n-1} y}{d x^{n-1}}+\cdots+\phi_{n} y=0
$$

which are, I believe, new, but there is one special case of them which belongs to the most classical part of the differential calculus. This is the case of the simplest equation of the form (1), that is

$$
\frac{d^{n} y}{d x^{n}}=0 \text {. }
$$

Denote the Wronskian

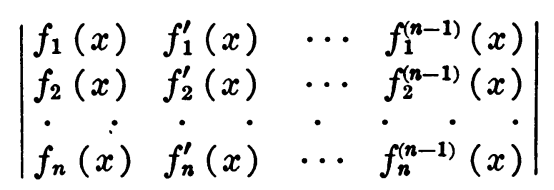

by $W\left(f_{1}(x), f_{2}(x), \cdots, f_{n}(x)\right)$. The equation (1) possesses in certain intervals the

Property $W$. There exist $n-1$ integrals $h_{1}(x), h_{2}(x), \cdots, h_{n-1}(x)$ of the homogeneous equation (1) satisfying the $n-1$ inequalities

(3) $h_{1}(x)>0, \quad W\left(h_{1}(x), h_{2}(x)\right)>0$, $\cdots, W\left(h_{1}(x), h_{2}(x), \cdots, h_{n-1}(x)\right)>0$

throughout the open intercal $(a, b)$.

Determine $n-1$ particular integrals of (1) by such initial conditions that the $n-1$ Wronskians in question are $=1$ for $x=x_{0}$; then the inequalities (3) are satisfied in a sufficiently small interval surrounding the point $x_{0}$.

* Presented to the Society, October 27, 1923. 
Equation (2) possesses the property $W$ in the interval $(-\infty,+\infty)$ since the functions $1, x, x^{2}, \cdots, x^{n-2}$ are integrals of (2) and we have

$$
1>0, W(1, x)=1 !>0, \quad W\left(1, x, x^{2}\right)=1 ! 2 !>0, \cdots .
$$

The equation

$$
y^{\prime \prime}+y=0
$$

is of the second order. Thus there is only one inequality (3), which is satisfied by the integral $\sin (x-a)$ for $a<x<a+\pi$. No integral of the equation (4) is $>0$ throughout an interval $(a, b)$ where $b-a>\pi$. Therefore the property $W$ belongs to (4) in any interval of length $\leqq \pi$ but in no interval of length $>\pi$. An equation $y^{\prime}+\phi y=0$ of the first order possesses the property $W$ in any interval where $\phi$ is continuous.

We assume now (and also in $\S \S 3-5$ ) the existence of the property $W$. Then we have the following theorems I, II, III:

Theorem I. If the function $f(x)$, supposed differentiable $n$ times, vanishes at $n+1$ points of the interval $(a, b)$, then there exists an intermediate point $\xi$ such that

$$
L f(\xi)=f^{(n)}(\xi)+\phi_{1}(\xi) f^{(-1)}(\xi)+\cdots+\phi_{n}(\xi) f(\xi)=0 .
$$

Theorem I.is a generalization of Rolle's theorem. Apply I to the equation (2) putting $n=1$; then we have the ordinary theorem of Rolle.

TheOREM II. There exists one and only one integral $H(x)$ of the homogeneous equation (1) assuming $n$ given values at $n$ given points of the interval $(a, b)$.

Theorem II asserts the possibility of a certain interpolation; in the case of equation (2) and if the $n$ points are distinct, it asserts the possibility of solving the problem which is solved explicitly by Lagrange's formula.

TheOREM III. Determine an integral $H(x)$ of the homogeneous equation (1) assuming the same values as $f(x)$ at $n$ given points of $(a, b)$; determine further an integral $N(x)$ of the non-homogeneous equation

$$
L y \equiv \frac{d^{n} y}{d x^{n}}+\phi_{1} \frac{d^{n-1} y}{d x^{n-1}}+\cdots+\phi_{n} y=1,
$$

that vanishes at the $n$ points in question. There exists a point $\xi$ intermediate between these $n$ points and an arbitrary point $x$ of $(a, b)$ such that

$$
f(x)=H(x)+N(x) L f(\xi) .
$$

Take the equation (2) and $n$ coincident points; in this particular case Theorem III gives Taylor's formula with Lagrange's remainder-term.

In all the theorems just stated coinciding points are admissible; this will be fully explained in $\S 1$. $\S 2$ enumerates some useful formulas concerning 
Wronskians. The proofs of Theorems I, II, III and a few illustrative examples are given in $\$ \S 3-5$. In $\S 6$ I try to justify the definition of property $W$ by proving the converse of Theorem II. $\$ 7$ deals with a system of several functions of one variable and generalizes Rolle's theorem in another manner.

1. The function $f(x)$ is supposed differentiable $n$ times. We say that $f(x)$ vanishes at $k$ points coinciding with $x_{0}$ if

$$
f\left(x_{0}\right)=f^{\prime}\left(x_{0}\right)=\cdots=f^{(k-1)}\left(x_{0}\right)=0 .
$$

This statement has a definite meaning if $k \leqq n+1$. If we say that $f(x)$ vanishes at $k$ and no more points coinciding with $x_{0}$, then we add to (6) the condition

$$
f^{(k)}\left(x_{0}\right) \neq 0,
$$

and we suppose $k \leqq n$. If $f(x)$ vanishes at

$$
m_{1}, m_{2}, m_{3}, \cdots, m_{l}
$$

points coinciding with

$$
x_{1}, x_{2}, x_{3}, \cdots, x_{l}
$$

respectively where

$$
a<x_{1}<x_{2}<x_{3}<\cdots<x_{l}<b
$$

then we say that $f(x)$ vanishes at $m_{1}+m_{2}+\cdots+m_{l}$ points of the interval $(a, b)$. If we say that $f(x)$ and $\phi(x)$ assume the same values at $n$ points of $(a, b)$ we mean that $f(x)-\phi(x)$ vanishes at $n$ points of $(a, b)$. Theorem II may be stated more explicitly thus: if there are given $l$ points (8) satisfying (9), $l$ positive integers (7) such that $m_{1}+m_{2}+\cdots+m_{l}=n$, and finally $l$ systems of values

$$
\begin{aligned}
& y_{1}, y_{1}^{\prime}, y_{1}^{\prime \prime}, \cdots, y_{1}^{\left(m_{1}-1\right)}, \\
& y_{2}, y_{2}^{\prime}, y_{2}^{\prime \prime}, \cdots, y_{2}^{\left(m_{2}-1\right)}, \\
& \cdot \quad \cdot \\
& y_{l}, y_{l}^{\prime}, y_{l}^{\prime \prime}, \cdots, y_{l}^{\left(m_{l}-1\right)},
\end{aligned}
$$

then there exists an integral $H(x)$ of (1) satisfying the conditions

$$
\begin{aligned}
& H\left(x_{1}\right)=y_{1}, \quad H^{\prime}\left(x_{1}\right)=y_{1}^{\prime}, \quad \cdots, \quad H^{\left(m_{1}-1\right)}\left(x_{1}\right)=y_{1}^{\left(m_{1}-1\right)}, \\
& H\left(x_{2}\right)=y_{2}, \quad H^{\prime}\left(x_{2}\right)=y_{2}^{\prime}, \quad \cdots, \quad H^{\left(m_{2}-1\right)}\left(x_{2}\right)=y_{2}^{\left(m_{2}-1\right)} \text {, } \\
& H\left(\dot{x_{l}}\right)=\dot{y}_{l}, \quad \dot{H^{\prime}}\left(\dot{x_{l}}\right)=\dot{y}_{l}^{\prime}, \quad \cdots, \quad H^{\left(m_{l}-1\right)}\left(x_{l}\right)=\dot{y_{l}^{\left(m_{l}-1\right)},}
\end{aligned}
$$

and $H(x)$ is completely determined by these conditions.

A point $\xi$ satisfying the inequality $x_{1}<\xi<x_{l}$ is said to be intermediate between the points (8); so far we have supposed $l>1$. If $l=1$, that is, if all the points in question coincide with one point $x_{1}$, then $\xi=x_{1}$ is the only intermediate point. 
I enumerate a few evident propositions used in what follows. The functions $u, u_{1}, \cdots, u_{k}$ are supposed continuous and differentiable as many times as required to calculate the expression

$$
\begin{aligned}
u_{k} & \frac{d}{d x}\left(u_{k-1} \frac{d}{d x}\left(u_{k-2} \cdots \frac{d}{d x}\left(u_{1} \frac{d}{d x}(u f)\right) \cdots\right)\right) \\
& =u_{k} \frac{d}{d x} u_{k-1} \frac{d}{d x} u_{k-2} \cdots \frac{d}{d x} u_{1} \frac{d}{d x} u f=u u_{1} u_{2} \cdots u_{k} \frac{d^{k} f}{d x^{k}}+\cdots
\end{aligned}
$$

1. If $f(x)$ vanishes at $k$ (and no more) points coinciding with $x_{0}$, then $f^{\prime}(x)$ vanishes at $k-1$ (and no more) points coinciding with $x_{0}$.

2. If $f(x)$ vanishes at $k$ points coinciding with $x_{0}$, then $u(x) f(x)$ vanishes at $k$ (or more) points coinciding with $x_{0}$.

3. Suppose $f(x)$ vanishes at $k$ and no more points coinciding with $x_{0}$, and suppose further that $u, u_{1}, u_{2}, \cdots, u_{k}$ are different from zero for $x=x_{0}$. Then the expression (11) is $\neq 0$ for $x=x_{0}$.

4. Suppose $f(x)$ vanishes at $k+1$ points. Then, between these $k+1$ points, there exists an intermediate point $\xi$ at which the expression (11) vanishes.

We obtain 4 by repeated application of Rolle's theorem and of Theorems $1,2$.

2. Let $c_{1}, c_{2}, \cdots, c_{n-1}$ be constants; then we have

$$
\begin{aligned}
W\left(f_{1}, f_{2}, \cdots, f_{n-1}, c_{1} f_{1}+c_{2} f_{2}+\cdots+\right. & \left.c_{n-1} f_{n-1}+f_{n}\right) \\
& =W\left(f_{1}, f_{2}, \cdots, f_{n-1}, f_{n}\right) .
\end{aligned}
$$

Suppose the function $u$ is differentiable $n-1$ times; then we have

$$
W\left(u f_{1}, u f_{2}, \cdots, u f_{n}\right)=u^{n} W\left(f_{1}, f_{2}, \cdots, f_{n}\right) .
$$

In particular put $u=1 / f_{1}$; we obtain

$$
W\left[\left(\frac{f_{2}}{f_{1}}\right)^{\prime},\left(\frac{f_{3}}{f_{1}}\right)^{\prime}, \ldots,\left(\frac{f_{n}}{f_{1}}\right)^{\prime}\right]=\frac{W\left(f_{1}, f_{2}, \cdots, f_{n}\right)}{f_{1}^{n}} .
$$

For the derivative of a Wronskian we have

$$
W^{\prime}\left(f_{1}, f_{2}, \cdots, f_{n}\right)=\left|f_{k}, f_{k}^{\prime}, \cdots, f_{k}^{(n-2)}, f_{k}^{(n)}\right|_{k=1,2, \cdots, n} .
$$

Using (14) we obtain by the usual formula for a minor of the adjoint determinant

$$
\begin{aligned}
& W\left(f_{1}, \cdots, f_{n-2}, f_{n-1}\right) W^{\prime}\left(f_{1}, \cdots, f_{n-2}, f_{n}\right) \\
& -W^{\prime}\left(f_{1}, \cdots, f_{n-2}, f_{n-1}\right) W\left(f_{1}, \cdots, f_{n-2}, f_{n}\right) \\
& =W\left(f_{1}, \cdots, f_{n-2}\right) W\left(f_{1}, \cdots, f_{n-2}, f_{n-1}, f_{n}\right) \text {, } \\
& \frac{d}{d x} \frac{W\left(f_{1}, \cdots, f_{n-2}, f_{n}\right)}{W\left(f_{1}, \cdots, f_{n-2}, f_{n-1}\right)} \\
& =\frac{W\left(f_{1}, \cdots, f_{n-2}\right) W\left(f_{1}, \cdots, f_{n-2}, f_{n-1}, f_{n}\right)}{\left(W\left(f_{1}, \cdots, f_{n-2}, f_{n-1}\right)\right)^{2}} .
\end{aligned}
$$


If $h_{1}(x), h_{2}(x), \cdots, h_{n}(x)$ are $n$ linearly independent integrals of (1), we have

$$
\begin{gathered}
L y \equiv \frac{W\left(h_{1}, h_{2}, \cdots, h_{n}, y\right)}{W\left(h_{1}, h_{2}, \cdots, h_{n}\right)}, \\
W\left(h_{1}, h_{2}, \cdots, h_{n}\right)=c e^{-\int \phi_{1}(x) d x},
\end{gathered}
$$

where $c$ is a constant. Put

$$
\begin{array}{rlrl}
y & =Y_{0}, & & W\left(h_{1}, y\right)=Y_{1}, \\
W\left(h_{1}, h_{2}, y\right) & =Y_{2}, & \cdots, \quad W\left(h_{1}, h_{2}, \cdots, h_{n}, y\right)=Y_{n}, \\
1 & =W_{0}, & & h_{1}=W_{1}, \\
W\left(h_{1}, h_{2}\right) & =W_{2}, & \cdots, \quad W\left(h_{1}, h_{2}, \cdots, h_{n}\right) \quad=W_{n} .
\end{array}
$$

Applying (15) we obtain

$$
\frac{d}{d x} \frac{Y_{0}}{W_{1}}=\frac{W_{0} Y_{1}}{W_{1}^{2}}, \quad \frac{d}{d x} \frac{Y_{1}}{W_{2}}=\frac{W_{1} Y_{2}}{W_{2}^{2}}, \quad \cdots, \quad \frac{d}{d x} \frac{Y_{n-1}}{W_{n}}=\frac{W_{n-1} Y_{n}}{W_{n}^{2}} .
$$

Combining these formulas with (16) we find finally

$$
L y \equiv \frac{W_{n}}{W_{n-1}} \frac{d}{d x} \frac{W_{n-1}^{2}}{W_{n-2} W_{n}} \cdots \frac{d}{d x} \frac{W_{2}^{2}}{W_{1} W_{3}} \frac{d}{d x} \frac{W_{1}^{2}}{W_{0} W_{2}} \frac{d}{d x} \frac{y}{W_{1}} .
$$

(18) is the usual decomposition of a linear homogeneous differential expression into a "product" of differential expressions of the first order. The proof here given is perhaps more direct than the usual.*

3. The property $W$ implies the non-vanishing of $W_{1}, W_{2}, \cdots, W_{n-1}$ appearing in formula (18). $W_{n}$ vanishes nowhere, by virtue of (17). Applying Theorem 4 of $\S 1$ to the decomposition (18) we get at once Theorem I.

Apply now Theorem I to $f(x)-H(x)$ instead of $f(x)$, where $H(x)$ is an integral of the homogeneous equation (1). Then $L H(x)=0$ and we obtain the corollary

THEOREM I*. If the function $f(x)$, supposed differentiable $n$ times, assumes at $n+1$ points of the interval $(a, b)$ the same values as an integral of the homogeneous equation (1), there exists an intermediate point $\xi$ such that

$$
L f(\xi)=f^{(n)}(\xi)+\phi_{1}(\xi) f^{(n-1)}(\xi)+\cdots+\phi_{n}(\xi) f(\xi)=0 .
$$

Theorem I* was incidentally remarked by $\mathrm{H}$. Poincaré $\dagger$ in the case of an equation of the second order where the property $W$ is particularly simple, including only one inequality. Poincaré's proof is very different from the proof given here and more complicated. I obtained the results presented in

* Cf. L. Schlesinger, Handbuch der Theorie der linearen Differentialgleichungen, vol. I, p. 52, formula (14).

† L'Intermédiaire des Mathématiciens, vol. 1 (1894), pp. 141-144 and also pp. $69,127,172,216$. 
this paper in clearing up and working out the original remark made by Poincaré. Another corollary to Theorem I is the following

Theorem I*** No integral $N(x)$ of the non-homogeneous equation (5) vanishes at more than $n$ points of the interval $(a, b)$.

Suppose, if possible, that $N(x)$ vanishes at $n+1$ points; then we have by Theorem I

$$
L N(\xi)=0
$$

at an intermediate point $\xi$; now, actually, $L N(\xi)=1$ and thus Theorem I*** is proved.

4. The general integral of (1) has the form

$$
H(x)=c_{1} h_{1}(x)+c_{2} h_{2}(x)+\cdots+c_{n} h_{n}(x),
$$

where $c_{1}, c_{2}, \cdots, c_{n}$ are constants. The conditions (10) form a system of $n$ linear non-homogeneous equations with the aid of which we have to determine $c_{1}, c_{2}, \cdots, c_{n}$. We have to prove that this system is neither incompatible nor indeterminate. Thus we have to prove that the only solution of the homogeneous system obtained from (10) by putting

$$
y_{1}=y_{1}^{\prime}=y_{2}^{\prime \prime}=\cdots=y_{l}^{\left(m_{l}-1\right)}=0
$$

is the identically vanishing solution.

Suppose, if possible, that there is a solution of the homogeneous system (10) (19) which does not vanish identically; denote it by

$$
H(x)=\gamma_{1} h_{1}(x)+\gamma_{2} h_{2}(x)+\cdots+\gamma_{k} h_{k}(x)+\cdots+\gamma_{n} h_{n}(x)
$$

where

$$
\gamma_{k} \neq 0, \quad \gamma_{k+1}=\gamma_{k+2}=\cdots=\gamma_{n}=0 .
$$

Then $h_{k}(x)$ assumes at $n \geqq(k-1)+1$ points the same values as an integral of the equation

$$
\frac{W\left(h_{1}, h_{2}, \cdots, h_{k-1}, y\right)}{W\left(h_{1}, h_{2}, \cdots, h_{k-1}\right)}=0
$$

of order $k-1$. Then there exists, by virtue of $I_{*}$, an intermediate point $\xi$ at which

$$
W\left(h_{1}(\xi), h_{2}(\xi), \cdots, h_{k-1}(\xi), h_{k}(\xi)\right)=0 .
$$

This last equation is excluded if we assume the existence of the property $W$ and thus Theorem II is proved.

Example. Assume that the algebraic equation

$$
x^{n}+c_{1} x^{n-1}+\cdots+c_{n}=0
$$

has $n$ different real roots $a_{1}, a_{2}, \cdots, a_{n}$, where

$$
a_{1}<a_{2}<a_{3}<\cdots<a_{n} .
$$


Then the linear homogeneous equation with constant coefficients

$$
y^{(n)}+c_{1} y^{(n-1)}+c_{2} y^{(n-2)}+\cdots+c_{n} y=0
$$

has the integrals

$$
e^{a_{1} x}, e^{a_{2} x}, \cdots, e^{a_{n} x},
$$

and possesses the property $W$ in the interval $(-\infty,+\infty)$ since the Wronskians

divided by

$$
e^{a_{1} x}, W\left(e^{a_{1} x}, e^{a_{2} x}\right), \cdots, W\left(e^{a_{1} x}, e^{a_{2} x}, \cdots, e^{a_{n} x}\right)
$$

$$
e^{a_{1} x}, e^{\left(a_{1}+a_{2}\right) x}, \cdots, e^{\left(a_{1}+a_{2}+\cdots+a_{n}\right) x}
$$

respectively reduce to positive constants which may be calculated by Vandermonde's formula. Therefore we can interpolate by a suitably chosen linear combination of the $n$ integrals (21) $n$ arbitrary values at the $n$ points $x_{1}, x_{2}$, $\cdots, x_{n}$ where

$$
x_{1}<x_{2}<x_{3}<\cdots<x_{n} .
$$

This fact is equivalent to the statement that

$$
\left|\begin{array}{lcll}
e^{a_{1} x_{1}} & e^{a_{1} x_{2}} & \cdots & e^{a_{1} x_{n}} \\
e^{a_{2} x_{1}} & e^{a_{2} x_{2}} & \cdots & e^{a_{2} x_{n}} \\
\cdot & \cdot & \cdot & \cdot \\
e^{a_{n} x_{1}} & e^{a_{n} x_{2}} & \cdots & e^{a_{n} x_{n}}
\end{array}\right| \neq 0 .
$$

Thus we have proved (23) as a particular case of Theorem II. It may be shown directly in a slightly different manner that the determinant (23) is $>0$ if (20) (22) are satisfied. I add that the property $W$ belongs to any equation whatever with constant coefficients in the interval $(-\infty,+\infty)$ if the roots of the corresponding algebraic equation are all real, whether distinct or not. We get a simple proof of this from (17).

Any integral $N(x)$ of the non-homogeneous equation (5) is of the form

$$
N(x)=N_{0}(x)+H(x) .
$$

Here $N_{0}(x)$ is a particular integral of the non-homogeneous equation (5) and $H(x)$ is the general integral of the homogeneous equation $(1) ; H(x)$ contains $n$ arbitrary constants which we can adapt to any conditions of the form (10), by virtue of Theorem II. Thus we obtain the corollary

Theorem II*. There exists one and only one integral $N(x)$ of the nonhomogeneous equation (5) assuming $n$ given values at $n$ given points of the interval $(a, b)$.

5. I now prove Theorem III. The possibility of determining $H(x)$ according to the enunciated conditions follows from Theorem II and the possibility 
of determining $N(x)$ follows from II*. Let $x_{0}$ be a point of $(a, b)$ but not one of the $n$ points mentioned at which $f(x)-H(x)$ and $N(x)$ vanish. Then we have, by Theorem I***, $N\left(x_{0}\right) \neq 0$. Therefore it is possible to find a constant $C$ satisfying the equation

$$
f\left(x_{0}\right)=H\left(x_{0}\right)+C N\left(x_{0}\right) .
$$

Thus the function

$$
f(x)-H(x)-C N(x)
$$

vanishes at $n+1$ points of $(a, b)$. There exists, by Theorem I, an intermediate point $\xi$ at which

that is,

$$
L(f(\xi)-H(\xi)-C N(\xi))=L f(\xi)-0-C \cdot 1=0,
$$

$$
C=L f(\xi) \text {. }
$$

Substituting this value in (24) we have Theorem III.*

I will now give some concrete cases of Theorem III with immediate applications.

Example 1. Let $x_{1}$ be the point at which we interpolate the differentiable function $f(x)$ by an integral of the equation

$$
y^{\prime}+y=0 \text {. }
$$

Adopting the notation of Theorem III we have

$$
H(x)=f\left(x_{1}\right) e^{x_{1}-x}, \quad N(x)=1-e^{x_{1}-x},
$$

and consequently for $x>x_{1}$

$$
f(x)=f\left(x_{1}\right) e^{x_{1}-x}+\left(1-e^{x_{1}-x}\right)\left(f(\xi)+f^{\prime}(\xi)\right)
$$

where $x_{1}<\xi<x$. From (25) we get at once the proposition $\dagger$

Suppose $f(x)$ differentiable for $x>0$; then

implies $\lim f(x)=c$.

$$
\lim _{x \rightarrow+\infty}\left(f(x)+f^{\prime}(x)\right)=c
$$

We may assume $c=0$ (and apply the proof to $f(x)-c$ instead of to $f(x)$ ). Choose first $x_{1}$ sufficiently great so that $\left|f(x)+f^{\prime}(x)\right|<\epsilon / 2$ for $x>x_{1}$; then choose $x_{2}, x_{2}>x_{1}$, such that $\left|f\left(x_{1}\right)\right| e^{x_{1}-x_{2}}<\epsilon / 2$. Finally we get by (25) for $x>x_{2}$

$$
|f(x)|<\frac{\epsilon}{2}+\frac{\epsilon}{2}
$$

\footnotetext{
* The particular case corresponding to (2) was given by Ch. Hermite for points coinciding in an arbitrary manner. See EEuvres, vol. III, pp. 432-443, and T. J. Stieltjes, EEuvres, vol. I, pp. 47-60. For a more general point of view see G. D. Birkhoff, these Transactions, vol. 7 (1906), pp. 107-136.

† G. H. Hardy, Quarterly Journal of Mathematics, vol. 35 (1903), pp. 31-33. The proof given by O. Perron, Mathematische Zeitschrift, vol. 6 (1920), p. 159, supposes more than the derivability of $f(x)$.
} 
By the same argument we may prove an analogous result for any equation with constant coefficients if the roots of the corresponding algebraic equation are real and negative.*

Example 2. In dealing with the equation

$$
y^{\prime \prime}+y=0
$$

we may choose for $(a, b)$ any interval of length $\pi$. The two points at which we interpolate may be different or not. Interpolating at two distinct points $x_{1}, x_{2}$, where

$$
x_{1}<x_{2}<x_{1}+\pi
$$

we find the formula

$$
\begin{aligned}
f(x)= & \frac{f\left(x_{1}\right) \sin \left(x_{2}-x\right)+f\left(x_{2}\right) \sin \left(x-x_{1}\right)}{\sin \left(x_{2}-x_{1}\right)} \\
& +\left(1-\frac{\cos \left(x-\frac{x_{1}+x_{2}}{2}\right)}{\cos \frac{x_{2}-x_{1}}{2}}\right)\left(f^{\prime \prime}(\xi)+f(\xi)\right)
\end{aligned}
$$

valid for $x_{2}-\pi<x<x_{1}+\pi ; \xi$ is an interior point of the least interval containing $x_{1}, x_{2}, x$. Interpolating at two points coinciding with $x_{1}$ we find

$$
\begin{aligned}
f(x)=f\left(x_{1}\right) \cos \left(x-x_{1}\right)+ & f^{\prime}\left(x_{1}\right) \sin \left(x-x_{1}\right) \\
& +\left(1-\cos \left(x-x_{1}\right)\right)\left(f^{\prime \prime}(\xi)+f(\xi)\right)
\end{aligned}
$$

valid for $x_{1}-\pi<x<x_{1}+\pi ; \xi$ lies between $x_{1}$ and $x$. We see that restriction (26) is essential for the validity of (27), $x_{2}=x_{1}+\pi$ being generally inadmissible; this point will be cleared up by Theorem IV.

In order to give an application suppose $f(x)$ is an integral of the equation

$$
y^{\prime \prime}+\phi(x) y=0 \text {, }
$$

where

$$
\phi(x)>1 \text {; }
$$

suppose further that $f\left(x_{1}\right)>0$. We have by (29)

$$
f^{\prime \prime}(\xi)+f(\xi)=-(\phi(\xi)-1) f(\xi) ;
$$

thus we may put (28) in the form

$$
\text { (31) } f(x)=A \sin (x-a)-\left(1-\cos \left(x-x_{1}\right)\right)(\phi(\xi)-1) f(\xi)
$$

*O. Perron, Mathematische Zeitschrift, vol. 6 (1920), pp. 161-163. 
where

$$
A \sin \left(x_{1}-a\right)=f\left(x_{1}\right)>0, \quad A \cos \left(x_{1}-a\right)=f^{\prime}\left(x_{1}\right) .
$$

The situation shown by (30) (31) (32) is this: the curve $A \sin (x-a)$ meets the curve $f(x)$ at the point $x_{1}$, see (32); the curve $A$ sin $(x-a)$ runs on the upper side of the curve $f(x)$ as far as $f(x)$ runs on the upper side of the $x$-axis, see (31) (30); but the curve $A \sin (x-a)$ cuts the $x$-axis at two points between which $x_{1}$ lies and whose distance apart is $\pi$; hence $f(x)$ reaches the $x$-axis necessarily before $A \sin (x-a)$ cuts it and we have the theorem

The distance between two consecutive zeros of any integral of the equation (29) satisfying the condition (30) is $<\pi$.

This is a particular case of a well known classical theorem given by Sturm* that can be proved generally by the argument I have used in the foregoing concrete case.

Any integral $f(x)$ of the equation (29) has the following property: the inequality

$$
f(x)>0
$$

implies

$$
f^{\prime \prime}(x)+f(x)<0 \text {. }
$$

Consider the curve of which the equation is

$$
r=\frac{1}{f(\vartheta)}
$$

in a system of polar coördinates $r, \vartheta$ with origin $O$. The inequality (34) expresses that the curve (35) appears from $O$ to be convex, as we may see, e.g., by (27). The result just proved may be formulated geometrically as follows:

$A$ curve appearing from a given point $O$ to be everywhere convex subtends an angle $<\pi$ at $O$.

H. Poincaré was led to the remark above mentioned $(\$ 3)$ by constructing an analytical demonstration of this geometrically evident fact.

6. The converse of Theorem II is also true: if the interpolation of any $n$ values by an integral of (1) is possible at any $n$ points of an interval, then the equation (1) possesses the property $W$ in that interval. The possibility of interpolation, that is, the existence of a solution of the non-homogeneous system (10) with arbitrary $y_{1}, y_{1}^{\prime}, \cdots, y_{l}^{\left(m_{l}-1\right)}$, is equivalent to the non-existence of a non-vanishing solution of the corresponding homogeneous system; we used this equivalence in $\S 4$. Thus the most simple form of the converse to Theorem II is the following:

* Cf. Maxime Bôcher, Méthodes de Sturm, Paris, 1917, p. 52. 
Theorem IV. If, except the identically vanishing integral, there exists no integral of equation (1) vanishing at $n$ points of the interval $a \leqq x<b$, then there exist $n-1$ integrals $h_{1}(x), h_{2}(x), \cdots, h_{n-1}(x)$ such that the Wronskians (36) $\quad h_{1}(x), W\left(h_{1}(x), h_{2}(x)\right), \cdots, W\left(h_{1}(x), h_{2}(x), \cdots, h_{n-1}(x)\right)$ vanish nowhere in the interval $a<x<b$.

The signs of the Wronskians are of no importance provided that they are constant. If the Wronskians (36) do not vanish we can make them positive by substituting $-h_{\nu}(x)$ for $h_{\nu}(x)$ for certain values of $\nu$.

Determine $n$ integrals $h_{1}(x), h_{2}(x), \cdots, h_{n}(x)$ satisfying the initial conditions

$$
\begin{aligned}
h_{1}(a) & =h_{1}^{\prime}(a)=h_{1}^{\prime \prime}(a)=\cdots=h_{1}^{(n-3)}(a)=h_{1}^{(n-2)}(a)=0, \\
h_{1}^{(n-1)}(a)=1, & \\
h_{2}(a) & =h_{2}^{\prime}(a)=h_{2}^{\prime \prime}(a)=\cdots=h_{2}^{(n-3)}(a)=0, h_{2}^{(n-2)}(a)=1, \\
h_{n-2}(a) & =h_{n-2}^{\prime}(a)=0, h_{n-2}^{\prime \prime}(a)=1, \\
h_{n-1}(a) & =0, \quad h_{n-1}^{\prime}(a)=1, \\
h_{n}(a) & =1 .
\end{aligned}
$$

These integrals are linearly independent because their Wronskian $= \pm 1$ for $x=a ; h_{1}(x)$ vanishes at $n-1$ points coinciding with $a$; by hypothesis there exists no $n$th point in the interval $a \leqq x<b$ at which $h_{1}(x)$ vanishes; therefore $h_{1}(x) \neq 0$ for $a<x<b$. Adopt the notation

$$
W\left(h_{1}, h_{2}, \cdots, h_{k}\right)=W_{k}
$$

that we used in $\S 2$ and suppose that we have already proved

$$
W_{1} \neq 0, \quad W_{2} \neq 0, \cdots, W_{k} \neq 0
$$

for $a<x<b, k<n$.

Let $c_{1}, c_{2}, \cdots, c_{k}$ be constants and put

$$
c_{1} h_{1}(x)+c_{2} h_{2}(x)+\cdots+c_{k} h_{k}(x)+h_{k+1}(x)=h(x) .
$$

$h(x)$ does not vanish identically, and therefore it vanishes at no more than $n-1$ points contained in the interval $a \leqq x<b$. Now $h(x)$ vanishes at $n-k-1$ points coinciding with $a$ by virtue of the conditions (37). Determine the constants $c_{1}, c_{2}, \cdots, c_{k}$ in such a manner that $h(x)$ vanishes at $k$ points coinciding with $x_{0}$ where $a<x_{0}<b$; this is possible because the determinant of the $k$ equations in question

$$
W\left(h_{1}\left(x_{0}\right), h_{2}\left(x_{0}\right), \cdots, h_{k}\left(x_{0}\right)\right) \neq 0,
$$

by the hypothesis of complete induction, that is, by (38). Beyond the

$$
(n-k-1)+k=n-1
$$


zeros already mentioned, $h(x)$ has no other zeros for $a \leqq x<b$. In particular $h(x)$ vanishes at no more than $k$ points coinciding with $x_{0}$. Consequently, by virtue of hypothesis (38) and of the Theorem 3 of $\S 1$,

$$
\begin{aligned}
& \frac{W_{k}^{2}}{W_{k-1}} \frac{d}{d x} \frac{W_{k-1}^{2}}{W_{k-2} W_{k}} \cdots \frac{d}{d x} \frac{W_{2}^{2}}{W_{1} W_{3}} \frac{d}{d x} \frac{W_{1}^{2}}{W_{0} W_{2}} \frac{d}{d x} \frac{h}{W_{1}} \\
& \quad=W\left(h_{1}, h_{2}, \cdots, h_{k}, h\right)=W\left(h_{1}, h_{2}, \cdots, h_{k}, h_{k+1}\right)=W_{k+1}
\end{aligned}
$$

does not vanish for $x=x_{0}$; we obtain (39) from (15) (12) in the same manner as we obtained (18). The point $x_{0}$ was arbitrarily chosen in the interval $a<x<b$ and thus Theorem IV is proved.

7. Another demonstration of the Theorems I, II, III essentially different from that given in $\$ \$ 1-5$ is based on the following theorem,

Theorem V. Suppose all the functions $h_{1}(x), h_{2}(x), \cdots, h_{n}(x), f(x)$ possess derivatives of order $n$ throughout the interval $a<x<b$ and satisfy the following $n$ inequalities:

$h_{1}(x)>0, \quad W\left(h_{1}(x), h_{2}(x)\right)>\underset{W\left(h_{1}(x), h_{2}(x), \cdots, h_{n}(x)\right)>0 .}{0}$.

Suppose also that the l points $x_{1}, x_{2}, \cdots, x_{l}$ and the corresponding positive integers $m_{1}, m_{2}, \cdots, m_{l}$ satisfy the conditions

$$
a<x_{1}<x_{2}<\cdots<x_{l}<b, \quad m_{1}+m_{2}+\cdots+m_{l}=n+1 .
$$

Consider the determinant of order $n+1$

$$
\begin{aligned}
\mid h_{k}\left(x_{1}\right), h_{\mathbf{k}}^{\prime}\left(x_{1}\right), & \cdots, h_{k}^{\left(m_{1}-1\right)}\left(x_{1}\right), h_{k}\left(x_{2}\right), h_{k}^{\prime}\left(x_{2}\right), \\
& \cdots, h_{k}^{\left(m_{2}-1\right)}\left(x_{2}\right), \cdots, h_{k}\left(x_{l}\right), \cdots, h_{k}^{\left(m_{l}-1\right)}\left(x_{l}\right) \mid,
\end{aligned}
$$

the $n$ first rows being obtained by putting successively $k=1,2,3, \cdots, n$ and the last row by substituting $f(x)$ for $h_{k}(x)$.

There exists an intermediate point $\xi, x_{1}<\xi<x_{l}$, such that the value assumed by the Wronskian at the point $\xi$, that is,

$$
W\left(h_{1}(\xi), h_{2}(\xi), \cdots, h_{n}(\xi), f(\xi)\right),
$$

is $>$, = or $<0$, according as the determinant $(40)$ is $>,=$ or $<0$.

Theorem V may be compared to a well known determinantal theorem of H. A. Schwarz.* Theorem V may be proved by complete induction; its validity for the $(n-1)+1$ functions

${ }^{*}$ H. A. Schwarz, Abhandlungen, vol. II, pp. 296-302; T. J. Stieltjes, EEuvres, vol. II, pp. 110-123. The determinant (40) has been considered by H. M. Morse, G. A. Pfeiffer, and G. M. Green from a different point of view; see Bulletin of the A merican Mathematical Society, vol. 23 (1916), pp. 114-122. 


$$
\left(\frac{h_{2}(x)}{h_{1}(x)}\right)^{\prime},\left(\frac{h_{3}(x)}{h_{1}(x)}\right)^{\prime}, \cdots,\left(\frac{h_{n}(x)}{h_{1}(x)}\right)^{\prime},\left(\frac{f(x)}{h_{1}(x)}\right)^{\prime}
$$

implies its validity for the $n+1$ functions $h_{1}(x), h_{2}(x), \cdots, h_{n}(x), f(x)$, as may be shown by (13) and by Rolle's theorem. I had originally based my demonstration of Theorems I, II, III on Theorem V. I was led to the treatment of the subject I finally adopted by a kind remark made by Professor $\mathrm{H}$. Weyl.

Eing. Techische Hochschule, Zurich, SwitzerLand.

\section{ERRATA, VOLUME 24}

J. F. RITT, On algebraic functions which can be expressed in terms of radicals. Page 21, lines 30 and 33, for " $n$ " read " $n$ ". 